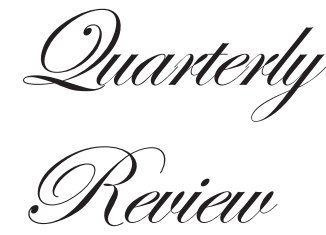

\title{
Radiological interventions in portal hypertension
}

\author{
Prasad Thottan Veedu, ${ }^{1}$ Shivanand Gamanagatti, ${ }^{1}$ Shalimar, ${ }^{2}$ Subrat \\ K Acharya ${ }^{2}$
}

\author{
ABSTRACT
}

Departments of Radiodiagnosis ${ }^{1}$ and Gastroenterology ${ }^{2}$

All India Institute of Medical Sciences New Delhi, India

Correspondence:

Dr. Shivanand Gamanagatti

Email:shiv223@rediffmail.com

The role of radiologists in the management of portal hypertension (PHT) had been confined to its diagnosis and treatment planning. However, with the advancements in the field of interventional radiology various minimally invasive techniques have been evolved for the management of portal hypertension and its complications such as recanalisation techniques, shunt creation and variceal bleeding control. Thus, with these technical advances, there is shift in the role of radiologist from diagnosis to management of portal hypertension. In this review, we discuss the nuances of various radiological interventions available for the treatment of portal hypertension.

KEYWORDS: portal hypertension, intervention radiology, recanalisation, shunt, variceal bleed

\section{Introduction}

Till recently the radiologist has had a restricted role in the management of portal hypertension. Conventionally radiology has been an aid to diagnosis and treatment planning for patients with portal hypertension (PHT). However with the arrival of new minimally invasive interventional radiology techniques, like recanalisation methods, shunt creation techniques and strategies for control of variceal bleeding, the role of radiologist is undergoing a paradigm shift, with a greater contribution of radiology in direct management of these patients.

\section{Portal hypertension}

Portal hypertension is characterized by abnormally high pressure gradient in the splanchnic circulation, which can be either due to increased vascular resistance or increased blood flow in the portal system. Increased resistance can occur broadly at three levels; pre-sinusoidal (e.g. portal vein thrombosis), sinusoidal (e.g. cirrhosis) or post-sinusoidal (e.g. Budd-Chiari syndrome). Increased portal blood flow as in cases of hypersplenism or arterio-portal shunts can also cause portal hypertension, rarely. The etiopathogenesis of portal hypertension varies in different age groups. In children the leading causes are extra hepatic portal venous obstruction (EHPVO) and Budd-Chiari syndrome (BCS $)^{1}$ whereas in adults, cirrhosis is the major cause (Table 1). ${ }^{2}$

The normal baseline portal pressure is between $7-10 \mathrm{mmHg}$ and the normal hepatic venous pressure gradient (HVPG) ranges from 1 to $4 \mathrm{mmHg}$. In portal hypertension the portal pressure rises above $10 \mathrm{mmHg}$ and the gradient exceeds 5 $\mathrm{mmHg}$. An HVPG above $10 \mathrm{mmHg}$ is associated with formation of esophageal varices and above $12 \mathrm{mmHg}$ augurs the risk of variceal bleeding and ascites. ${ }^{3}$ Portal hypertension can lead to life threatening variceal hemorrhage or development of morbid ascites and encephalopathy. Advanced stages of portal hypertension secondary to cirrhosis, can get complicated by hepato-renal syndrome carrying drastically high mortality rate. Severity of portal hypertension (baseline HVPG) is reported as an independent risk factor for the development of hepatocellular carcinoma in cirrhotic patients. ${ }^{4}$ The management of portal hypertension and its complications requires multi-modality 
Table 1: Etiology of portal hypertension

Prehepatic PHT (normal WHVP and FHVP with normal HVPG)*

- Portal vein thrombosis

- Splenic vein thrombosis

- Congestive splenomegaly (Banti's syndrome)

- Arteriovenous fistula

Hepatic PHT (increased WHVP, normal FHVP and increased HVPG)

1. Presinusoidal

- Schistosomiasis

- Congenital hepatic fibrosis

2. Sinusoidal

- Cirrhosis - (many causes)

- Alcoholic hepatitis

- Nodular regenerative hyperplasia

- Polycystic liver disease

3. Postsinusoidal

- Sinusoidal obstructive syndrome

- Budd-Chiari syndrome

Posthepatic PHT (increased WHVP and FHVP with normal HVPG)

- Inferior vena cava webs, thrombosis

- Cardiac causes (restrictive cardiomyopathy, constrictive pericarditis, and congestive heart failure)

- Pulmonary hypertension

*WHVP: wedged hepatic venous pressure; FHVP: free hepatic venous pressure; HVPG: hepatic venous pressure gradient

approach including medical and endoscopic management and surgical and nonsurgical methods (minimally invasive radiological techniques) to reduce portal pressure. Surgical methods carry significant morbidity and mortality in these patients.

\section{Interventions in portal hypertension}

The primary aim isto reduce the portal pressure and thereby reduce the complications related to PHT such as variceal bleed, ascites, hydrothorax and hepato-renal syndrome. The secondary aim, in cases where it is difficult to bring down the portal pressure, is to deal with the complications of PHT such as esophageal and gastric varices, refractory ascites and encephalopathy.The radiologic interventions available for PHT are broadly categorized in Table 2.

\section{Transjugular intrahepatic portosystemic shunt (TIPS)}

TIPS involves the creation of a low resistance conduit between the portal vein and hepatic vein by deploying an intra-hepatic stent. The procedure is carried out under fluoroscopic and
Table 2: Radiological interventions available for portal hypertension
Interventions that reduce portal blood pressure
a) Transjugular intrahepatic portosystemic shunts (TIPS)
b) Recanalisation of hepatic venous outflow
c) Partial splenic embolisation
d) Recanalisation of occluded/thrombosed portal veins or tributaries
e) Occlusion of arterio-portal shunts (congenital/acquired)
f) Revision of occluded surgical or radiological shunts
Interventions to control the symptoms of PHT
a) Percutaneous transhepatic variceal embolisation
b) Balloon occluded retrograded transvenous obliteration of gastric varices (BRTO)
Occlusion of surgical or radiologic shunts in refractory encephalopathy

ultrasound guidance, under conscious sedation or general anesthesia depending upon the patients' condition. Prophylactic antibiotics may be given and coagulation defects have to be corrected prior to the procedure.

Technique: The procedure starts with puncture of right internal jugular vein for venous access under sonographic guidance. A $10 \mathrm{~F}$ long vascular sheath is placed across the right heart into the inferior vena cava. An initial cavogram is obtained to check the inferior vena cava and the ostium of hepatic vein. A diagnostic angiographic catheter is then introduced into one of the hepatic veins(preferably right hepatic vein). At this stage, the baseline hepatic vein wedge pressure is measured using a balloon catheter occluding the hepatic vein outflow. After getting access into the right hepatic vein, this catheter is exchanged with a long metallic cannula with $7 \mathrm{~F}$ vascular sheath over the metallic guide wire. This metallic cannula is then wedged against the wall of hepatic vein close to the ostium and a gentle contrast study is carried out to look for retrograde visualization of intrahepatic portal vein branches. USG or direct transhepatic portography may also be used to visualize the portal vein and its branches in difficult cases with distorted anatomy. Then using a combination of a coaxial system of $16 \mathrm{G}$ metallic cannula with $7 \mathrm{~F}$ sheath and $21 \mathrm{G}$ Chiba needle with $4 \mathrm{~F}$ catheter,the hepatic parenchyma is entered through the wall of hepatic vein. Using either fluoroscopic landmark of portal vein or USG guidance, the portal vein is accessedusing a $21 \mathrm{G}$ Chiba needle with $4 \mathrm{~F}$ catheter loaded over it. This is the most critical step of TIPS where utmost care should be taken not to breach the liver capsule which can lead to life threatening intraperitoneal bleed. The preferable site of portal vein puncture is at the portal vein bifurcation or within 2 $\mathrm{cm}$ of the bifurcation. Once the portal vein has been entered, a portogram is obtained to examine the splenoportal axis anatomy 
and variceal status. The parenchymal tract is dilated using angiographic balloon catheters (diameter 8-10 mm) and a specially designed TIPS stent (uncovered segment in portal vein and covered segment in hepatic parenchyma and hepatic vein) is deployed to maintain the patency of the tract. PTFE (polytetraflouroethylene)covered stents are preferred over bare stents as they offer better long term shunt patency and prevent porto-biliary fistula. ${ }^{5-7}$ The customized TIPS stent graft has a small uncovered part $(2 \mathrm{~cm})$ which is to be kept in the portal vein to promote good inflow and avoid portal vein thrombosis

\section{(Figure 1).}

The established indication for TIPS is in the management of patients with cirrhosis and portal hypertension complicated by recurrent/refractory variceal bleeding and refractory ascites. Refractory acutely bleeding varices and complicated acute or chronic Budd-Chiari syndrome are among other indications (Table 3). ${ }^{8}$ Absolute contraindications for TIPS placement include congestive cardiac failure, severe pulmonary hypertension, unrelieved biliary obstruction and preexisting hepatic encephalopathy (Table 4). ${ }^{9}$ Variceal bleeding is a major cause of death in cirrhotic patients and correlates with portal

\section{Table 3: Indications for TIPS}

- Secondary prevention of variceal bleeding*

- Refractory cirrhotic ascites*

- Refractory acutely bleeding varices

- Portal hypertensive gastropathy

- Bleeding gastric varices

- Refractory hepatic hydrothorax

- Hepato-renal syndrome

- Budd Chiari syndrome

- Veno-occlusive disease

- Hepato-pulmonary syndrome

*Efficacy determined by controlled trials

\section{Table 4: Contraindications for TIPS}

\section{Absolute}

- Right sided heart failure

- Biliary tract obstruction

- Uncontrolled infection

- Pulmonary hypertension

- Chronic recurrent disabling hepatic encephalopathy

- Hepatocellular carcinoma involving hepatic veins

Relative

- Severe liver failure (Pugh score $>12$ )

- Portal vein thrombosis

- Multiple hepatic cysts

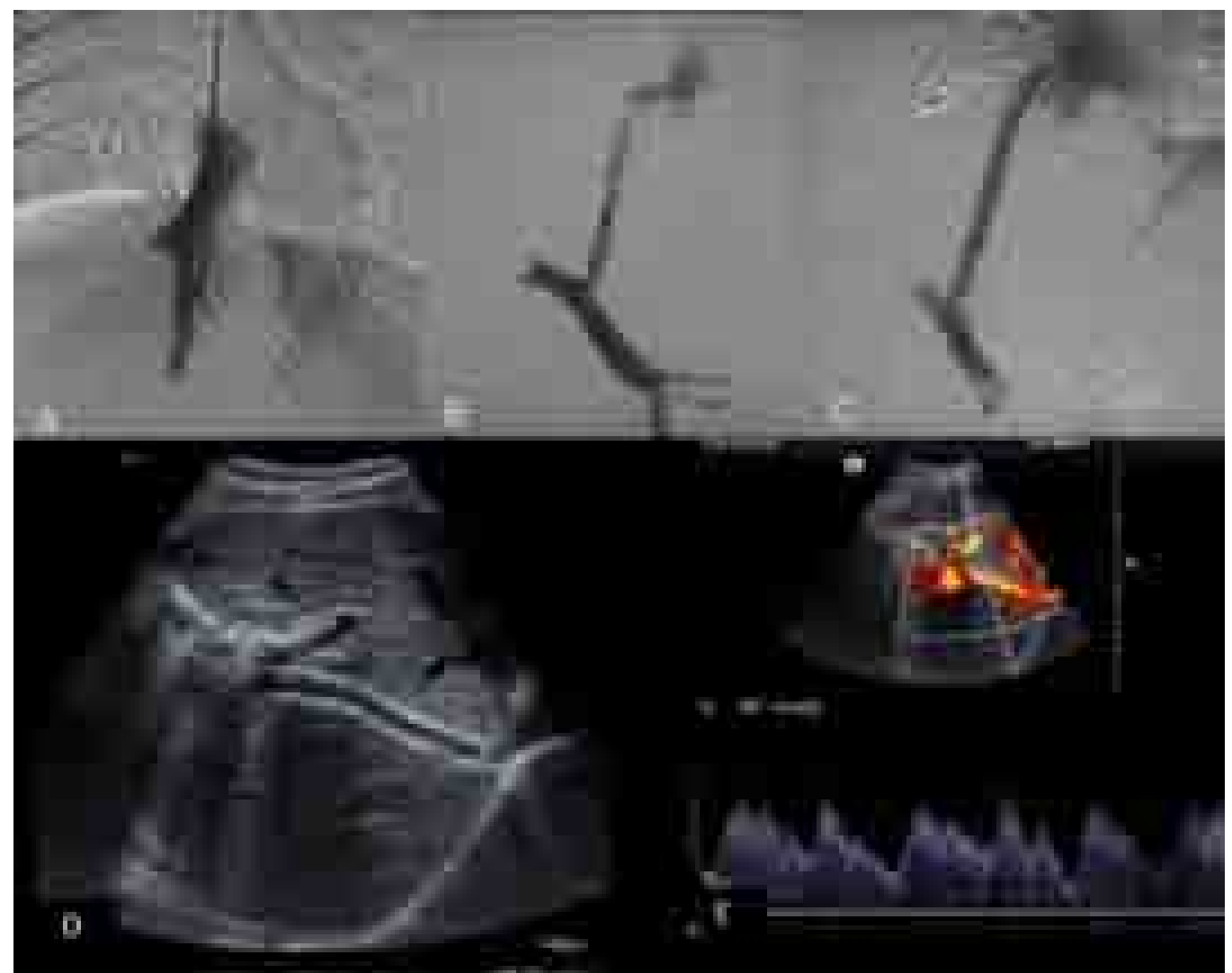

Figure 1: TIPS performed in a cirrhotic patient with uncontrolled variceal bleeding: A) initial cavogram showing normal caliber IVC and hepatic vein, B) portogram done after accessing the portal vein from the hepatic vein through liver parenchyma, C) post-TIPS venogram showing free flow of contrast through the stent graft, D\&E) USG and Doppler showing the TIPS stent with optimal flow 
hypertension. Many studies have demonstrated the efficacy of TIPS in controlling and preventing massive life threatening variceal hemorrhage. It is recommended for reducing the HVPG below $12 \mathrm{mmHg}$ for controlling variceal bleeding. TIPS is also beneficial in preventing bleeding due to hypertensive gastropathy. ${ }^{9}$ Refractory ascites reflects severe portal hypertension and advanced liver dysfunction and is associated with very poor outcome and survival. TIPS decreases the need for repeated large volume paracentesis in patients with refractory ascites. However in view of poor functional status of such patients and the higher risk of hepatic encephalopathy, TIPS is recommended for those who cannot tolerate repeated paracentesis. Similarly in cases of refractory hepatic hydrothorax, TIPS is recommended only for those whose effusion cannot be controlled with diuretics and sodium restriction. In cases of Budd-Chiari syndrome TIPS or DIPS (direct intrahepatic porto-systemic shunt) is recommended in cases of moderate severity and those who fail to respond to anticoagulation. $^{8}$

\section{Complications of TIPS}

Immediate complications are procedure related; such as hematoma, arrhythmia, stent displacement, bilhemia and shunt thrombosis. Uncommon life threatening complications include hemoperitoneum, cardiac failure, liver ischemia and sepsis. Chronic complications include congestive cardiac failure, portal vein thrombosis, chronic recurrent encephalopathy, TIPSitis and TIPS dysfunction. TIPS dysfunction is defined as loss of decompression of portal system due to occlusion or stenosis of the shunt. The best indicator of TIPS dysfunction is the reappearance of variceal bleeding and ascites, and can be demonstrated by imaging.

\section{Recanalisation of hepatic venous outflow (Budd- Chiari Syndrome)}

Budd-Chiari Syndrome (BCS) is a heterogeneous group of disorders characterized by hepatic venous obstruction at the level of hepatic veins, inferior vena cava (IVC) or right atrium. Hepatic venous outflow increases sinusoidal pressure, portal hypertension and decreased liver perfusion which in turn leads to progressive liver failure. The manifestations can range from fulminant acute liver failure to chronic liver disease depending on extent and acuteness of obstruction and adequacy of remaining collateral circulation. The etiology of obstruction in western population is primarily thrombosis (bland or tumor). Membranous web like obstruction of hepatic veins and IVC is commonly seen in Asian population.Medical therapy including anticoagulation for preventing recurrence and extension of thrombosis, and measures to control ascites and gastrointestinal bleeding,yield poor long term outcomes. Surgical options include creation of a porto-systemic shunt and liver transplant. Porto-systemic surgical shunts can only partially decompress the portal system and the feasibility liver transplant depends largely on availability of donor.

Percutaneous techniques for BCS include transluminal balloon angioplasty of hepatic veins and or IVC and stent placement. Percutaneous interventions can restore physiologic hepatic venous outflow and help in resolution of venous congestion, improvement in hepatic function and arresting hepatic destruction. Hepatic venous recanalisation techniques are feasible if the occlusion comprises a short segment with large patent segment. The transjugular approach is preferred for approaching the hepatic veins. Once the hepatic venous ostium is accessed, attempt is made to negotiate a guidewire across the stenosis followed by balloon angioplasty of the narrowed segment. Thetransjugular approach may fail in cases with tight stenosis at the ostium. The percutaneous transhepatic approach is preferred is such cases which involves USG guided puncture of the patent segment of hepatic veins and negotiating the guidewire across the stenosis into the IVC. The guidewire is then snared through the transjugular route and the procedure is completed via the transjugular route to avoid making larger tracts in the liver. Successful angioplasty is confirmed bythe free flow of contrast across the stenosis, reduction in pressure gradients and collapse of intrahepatic veno-venous collaterals (Figure 2). Metallic stents are deployed in cases with insufficient response orrapid re-stenosis following balloon angioplasty (Figure 3).Good midterm outcome is noted following angioplasty, provided adequate anticoagulation is maintained. ${ }^{10,11}$

IVC stenosis secondary to liver congestion in BCS get resolved after successful venous decompression procedures. However primary caval stenosis that occludes hepatic venous outflow has to be addressed by IVC angioplasty. IVC angioplasty is performed by femoral or jugular approach (Figure 4). Caval stenting is needed if angioplasty fails. Long segment occlusion of hepatic veins is difficult to reopen and have higher chances of re-occlusion and these patients require creation of a porto-systemic shunt. TIPS and DIPS are being increasingly performed in such scenario with gratifying results. ${ }^{12,13}$ 


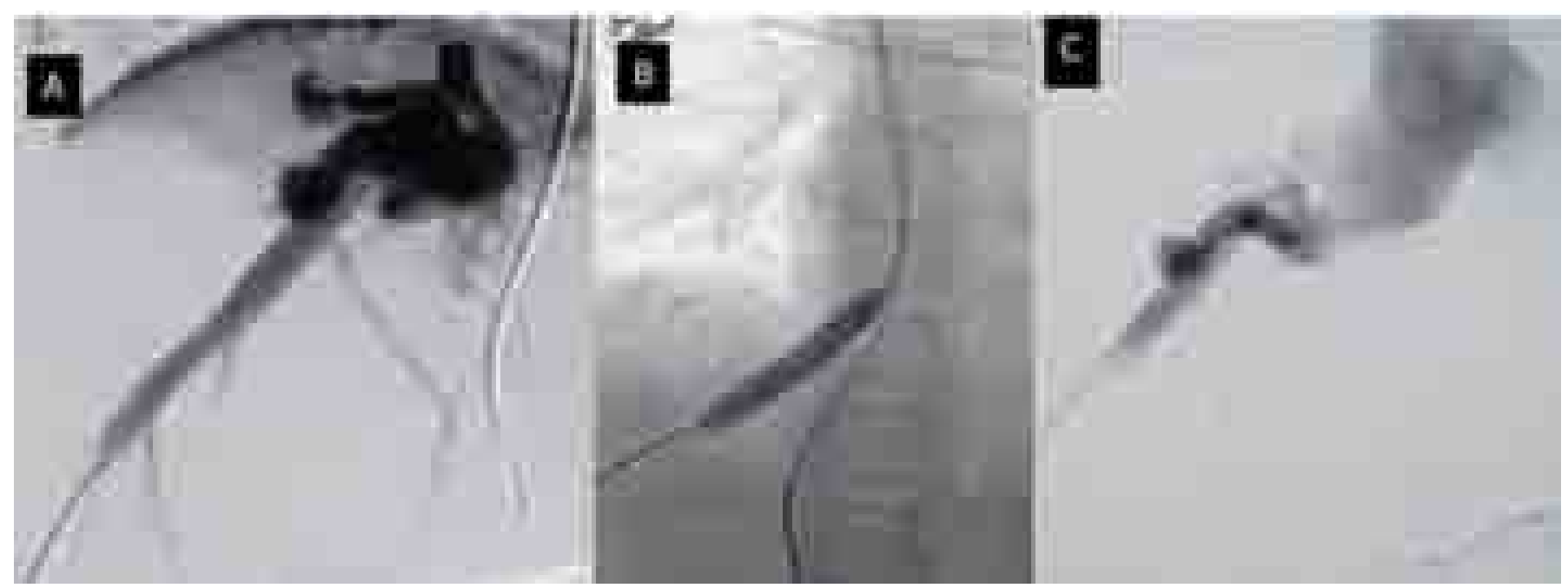

Figure 2: Hepatic vein angioplasty in a patient with Budd-Chiari syndrome: A) hepatic venogram after percutaneous approach shows complete occlusion at the ostium with multiple venous collaterals, B) balloon angioplasty of the stenosed ostium, C) postangioplasty hepatic venogram showing free flow of contrast into the IVC

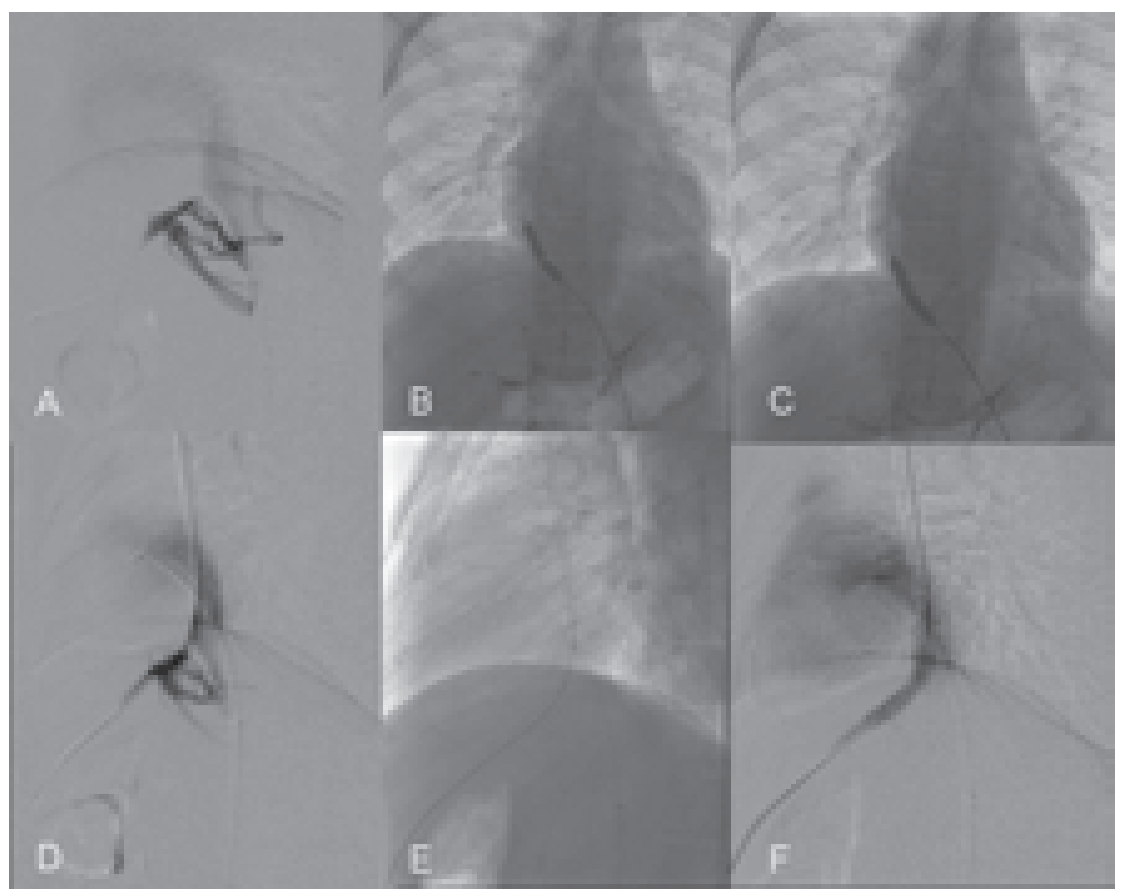

Figure 3: Hepatic vein stenting in a case of recurrent HVOTO: A) hepatic venogram showing stenosis at ostium with multiple collaterals, B \&C) balloon angioplasty, D) post-angioplasty image showing residual stenosis with persistent collaterals, E \&F) stent deployment and subsequent study showing free flow of contrast with disappearance of collaterals

\section{Partial splenic embolisation (PSE)}

PSE is performed to decrease portal venous inflow, thereby decreasing the portal pressure. It is performed by superselective catheterization of splenic arterial branches and embolisation using polyvinyl alcohol particles (Figure 5). PSE is effective in reducing episodes of variceal bleeding. It also helps in decreasing splenic size and in improving hematologic parameters and hepatic protein synthesis. ${ }^{14} \mathrm{PSE}$ is a safe, simple and effective alternative for splenectomy in hypersplenism. ${ }^{15}$
It causes partial necrosis of splenic parenchyma with minimal risk of complications such as liver abscess and sepsis. Splenic artery embolisation can also be used in management of bleeding gastric varices secondary to splenic vein thrombosis. ${ }^{16}$

\section{Recanalisation of occluded/thrombosed portal vein or its tributaries}

Portal hypertension can be caused by obstruction of portal vein or its tributaries. It can be due to inflammatory stenosis, a 


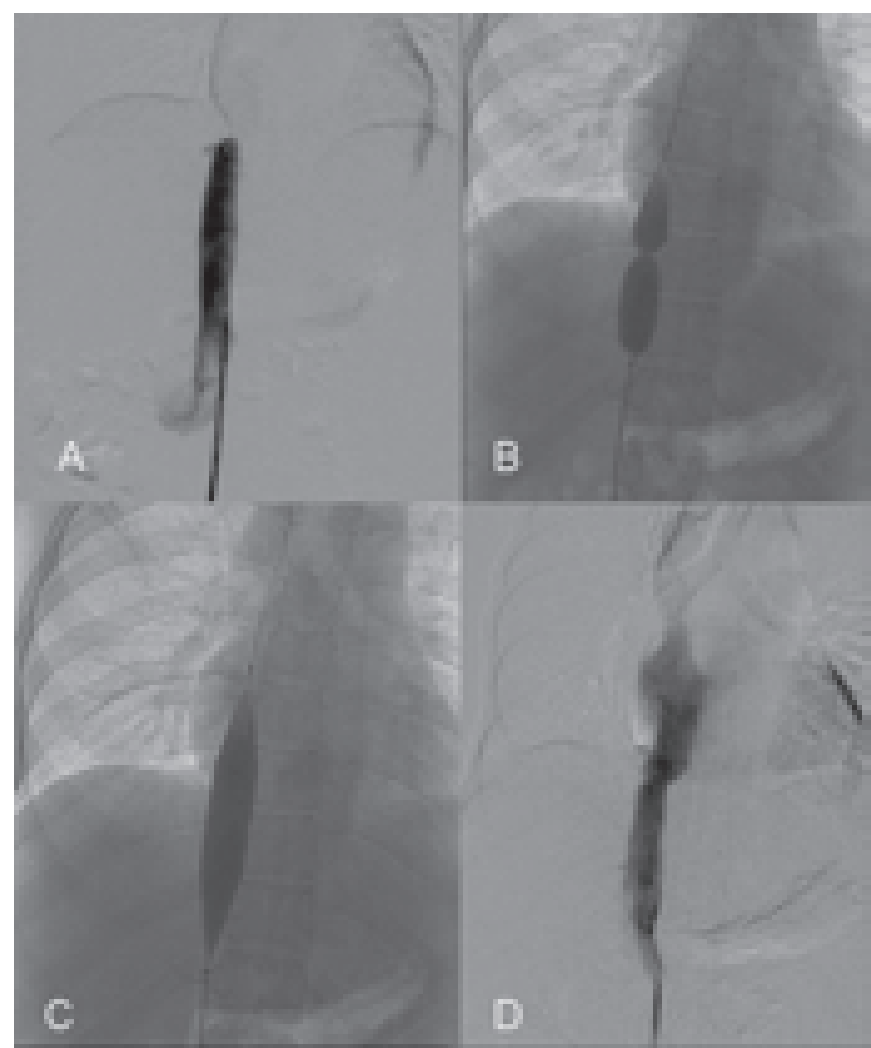

Figure 4: IVC angioplasty: A) cavogram showing total occlusion below cavo-atrial junction, B \&C) angioplasty using balloon catheter, D) post-angioplasty cavogram showing free flow of contrast into the right atrium
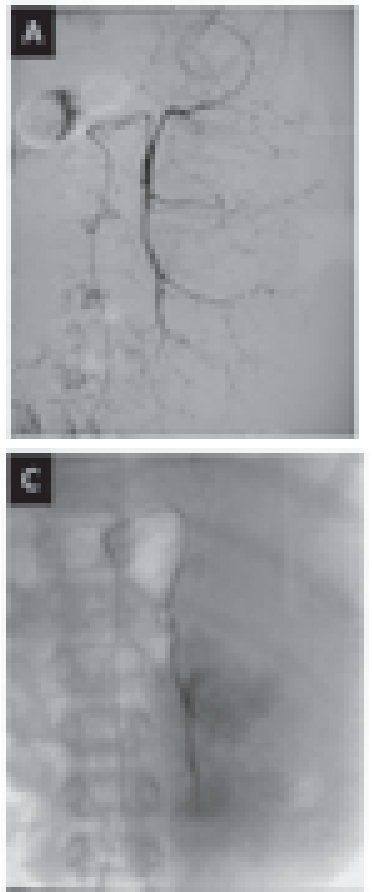
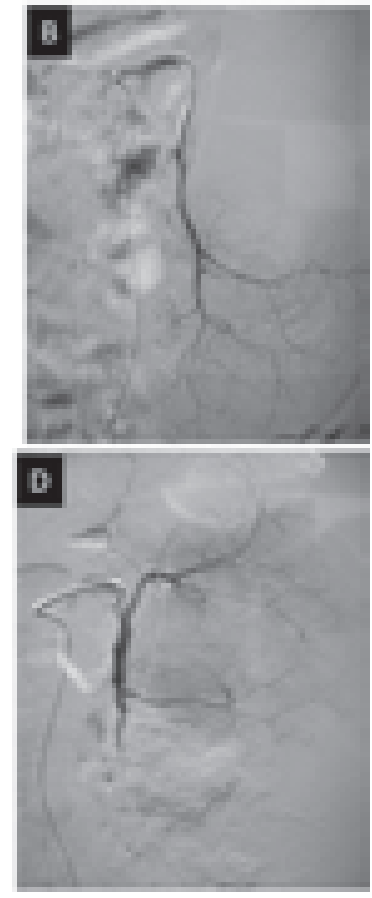

Figure 5: Partial splenic embolisation in a patient with hypersplenism: A) splenic arteriogram showing parenchymal branches, B) selective run of lower branch of splenic artery, C) embolisation of lower part of the spleen using PVA particles, D) post-embolisation study showing decreased perfusion in lower part bland thrombus or tumor infiltration. In both benign and malignant conditions patients present with variceal bleeding, ascites and/or abdominal pain. These symptoms can be relieved by recanalisation of occluded vessels which can be achieved by angioplasty and stenting through the transjugular, transhepatic or transplenic routes. ${ }^{17,18}$ Anastomotic strictures of portal vein can also be treated by angioplasty (Figure 6).

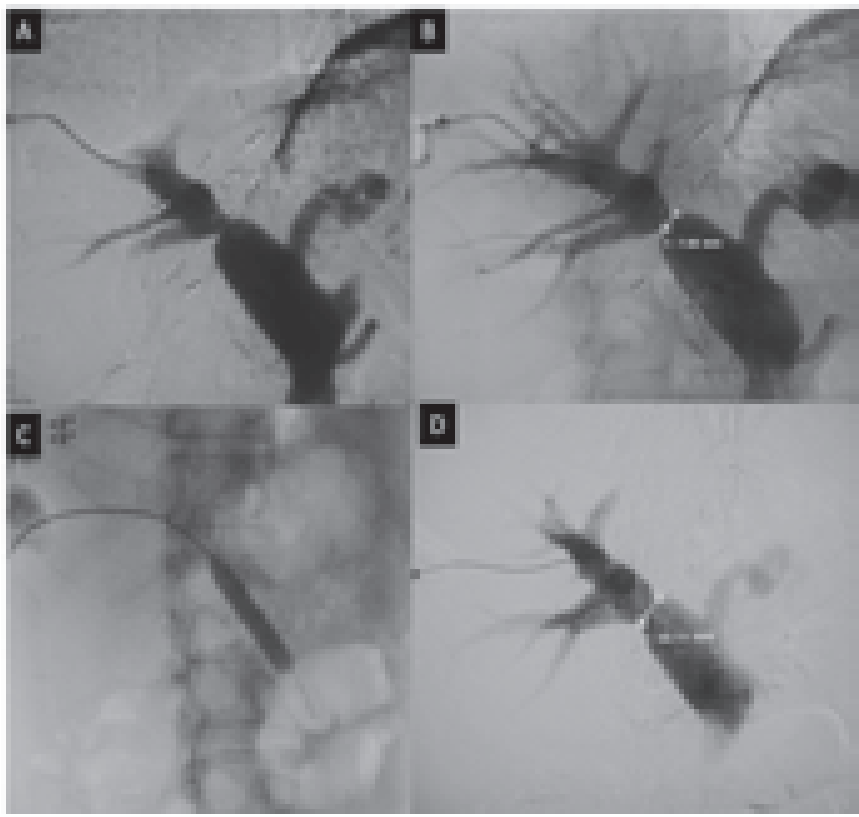

Figure 6: Recanalisation of the anastomotic stricture of portal vein: $A \& B$ ) portogram showing stricture at anastomotic site,C) balloon dilatation of the stricture,D) postangioplasty run showing improvement in stricture

\section{Occlusion of arterio-portal shunts (congenital/ acquired)}

Arterio-portal shunts can cause portal hypertension by increased portal flow. The etiology of arterio-portal shunts can be congenital malformation, trauma, tumors, aneurysms and surgery or hepatic interventions. Such patients may rarely present with gastrointestinal bleeding, ascites, heart failure and splenomegaly. Transarterial embolisation of feeding arteries by coils, glue or other closure devises, depending on the size of the fistula, is the preferred treatment in such cases ${ }^{19}$ (Figure 7).

\section{Revision of occluded surgical or radiological shunts}

Surgically created portosystemic shunts for portal hypertension may be complicated by thrombosis and occlusion. Surgical treatment of such cases is difficult and carries high morbidity. Occluded leno-renal shunts and mesocaval shunts can be 


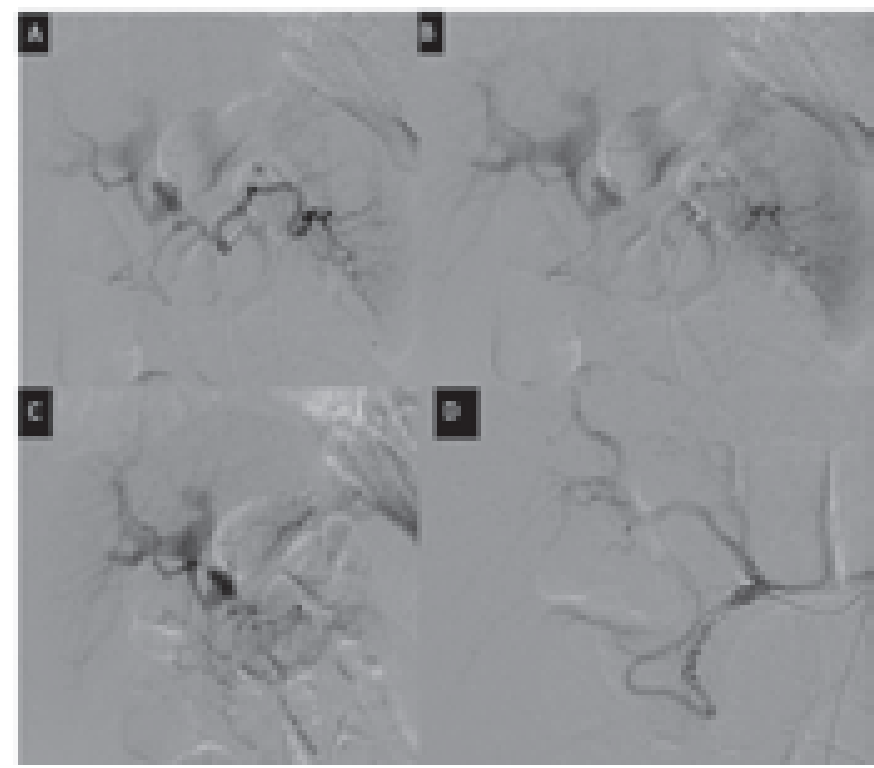

Figure 7: Occlusion of arterio-portal shunts (acquired):A\&B)celiac angiogram showing pseudoaneurysm of gastroduodenal artery communicating with portal vein,C) selective hepatic angiogram prior to embolisation showing pseudoaneurysm,D) post-embolisation study showing complete isolation of pseudoaneurysm and abnormal shunt

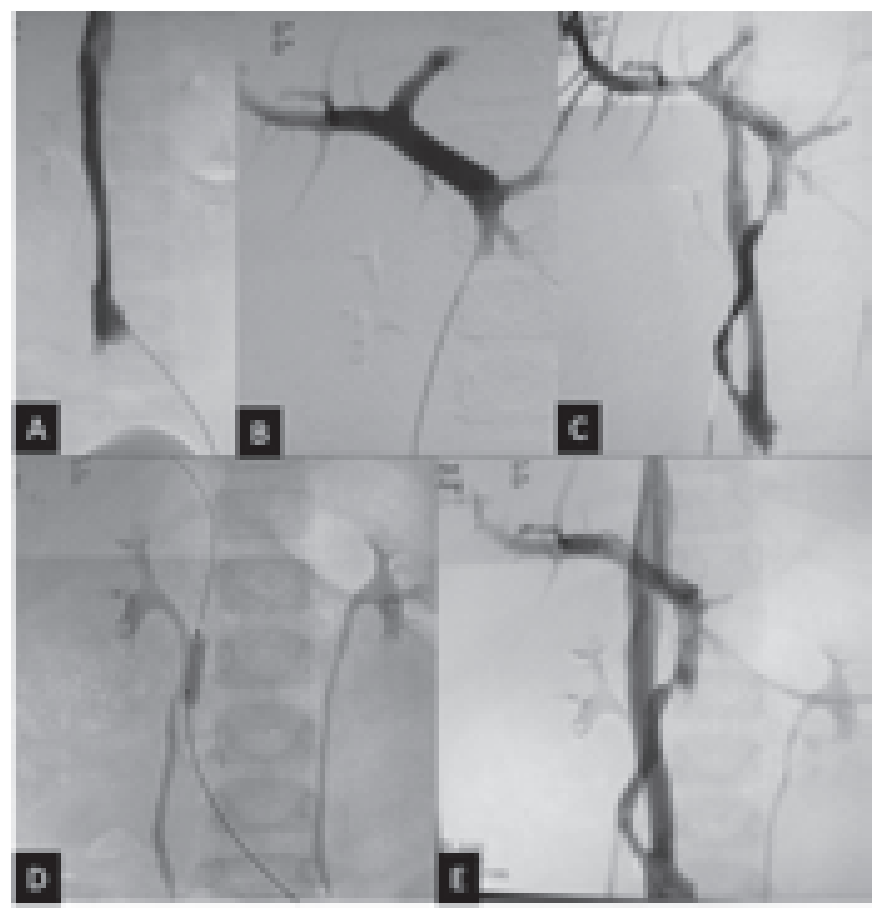

Figure 8: Mesocaval shunt revision:A) cavogram showing normal IVC, B\&C)portogram performed after advancing the catheter into the portal vein through meso-caval shunt showing stenosis in the shunt, D) balloon dilatation of stenosis, E) subsequent portogram showing improved flow of contrast through the shunt

successfully recanalised via theendovascular route (Figure 8). ${ }^{20,21}$

\section{Percutaneous transhepatic (or trans-splenic) variceal embolisation}

Percutaneous transhepatic variceal embolisation was introduced as a technique to manage bleeding esophago-gastric varices by Lunderquist and Vang in $1974 .{ }^{22}$ Subsequent studies had shown excellent immediate success rate of 70-90\%. A recurrent bleeding rate of 30-65\% was noted in these studies since the procedure alone did not address the basic issues of portal hypertension and hepatic insufficiency. ${ }^{23-27}$ Subsequently the procedure was recommended in acute settings when the variceal bleeding was not controlled by endoscopic sclerotherapy, vasopressin infusion and balloon tamponade. ${ }^{28}$ It can also be used for the management of bleeding gastric varices which are difficult to localize by endoscopy. ${ }^{29,30}$

The technique starts with percutaneous transhepatic puncture of the intrahepatic branch of portal vein with ultrasound or flouroguidance and advancement of the catheter into the splenic vein. Diagnostic spleno-portography is then performed to delineate the feeding veins, varices and draining veins. The common veins feeding the gastric varices are the left gastric, short gastric and posterior gastric veins. Subsequently the feeding vein is selectively catheterized and venography is performed to assess the size of the varices, velocity of flow and the size of gastrorenal shunt. On the basis of these data variceal sclerotherapy is performed with cyanoacrylate mixed with lipiodol. In cases with large gastrorenal shunts with rapid venous drainage the gastrorenal shunt has to be occluded with angiographic balloon catheter prior to sclerotherapy. The track in the hepatic parenchyma is embolised using gelfoam and steel coils at the end of the procedure (Figure 9). Variceal embolisation can also be done via the transplenic route, in which the access into portal system is through the splenic vein with rest of the procedure being the same. The transplenic route is especially helpful in cases with preexisting portal vein thrombosis. ${ }^{31}$

\section{Balloon occluded retrograded transvenous obliteration of gastric varices (BRTO)}

Patients of portal hypertension are often complicated by bleeding from esophago-gastric varices. Although less common, gastric variceal rupture often leads to profuse bleeding and are difficult to manage with endoscopic and surgical techniques. ${ }^{32}$ BRTO is a novel technique for the management 


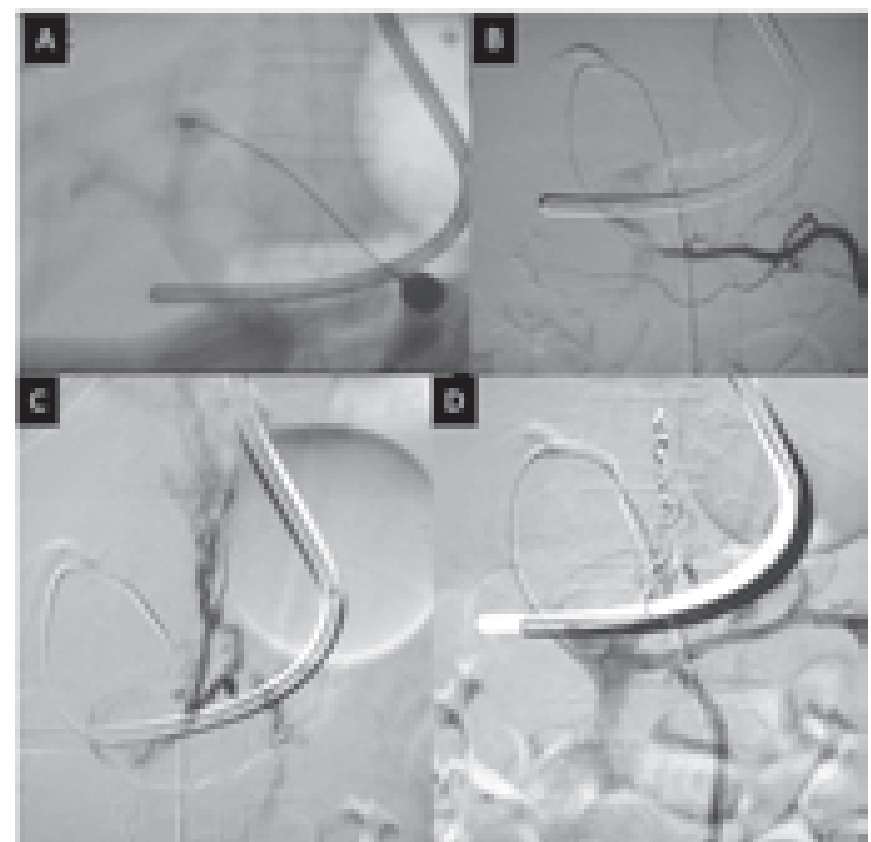

Figure 9: Percutaneous transhepatic variceal embolisation:A) percutaneous transhepatic portogram showing portal vein and branches,B) selective angiogram of coronary vein is showing gastric varices, C) feeding vein is managed by deploying stainless steel coils

of gastric varices with major gastro-systemic shunts. It involves obliteration of gastric varices using sclerosants after occluding the communicating shunts with an angiographic balloon catheter. Contrast enhanced computed tomography (CECT) of the abdomen is commonly performed for planning the procedure. The diameter of major shunts is assessed using CECT to calculate the size of balloon catheter which can occlude it. Gastro-renal shunt is commonly encountered. Other shunts such as gastro-caval and gastro-pericardiophrenic are also rarely seen.

The procedure is commonly performed with an access through the right femoral vein. After cannulating the left renal vein, guide wire is advanced into the left adrenal vein which is a common channel for gastro-renal shunt. The balloon catheter is advanced into the shunt over the guide wire and inflated so as to block the shunt. A contrast study is carried out through the catheter to see the opacification of gastric varices. Some of the varices may show leaking collaterals which can be dealt by deploying microcoils or gel foam pledgets after selective catheterisation. Subsequently, a sclerosant mixed with contrast (e.g. 5\% ethanolamine oleate with lipiodol or sodium tetradecyl sulphate with contrast medium like iomeron) is injected into the varices through the balloon catheter. The angiographic balloon is kept inflated for a variable time ranging from one to 24 hours for adequate thrombosis to occur in the varices. After allowing time for thrombi formation, the hemolysed blood with the remaining sclerosant is aspirated,the balloon is deflated and catheter removed. If free flow of blood is seen with the aspirate, supplemental dose of sclerosant can be injected. Amount of sclerosant and duration of application is up to the discretion of the radiologist and it varies with the size and extent of varices. Rarely varices may show multiple shunts and balloon occlusion of all the shunts would be required in such cases. CT scan is performed after 2-4 days to gauge immediate response. Follow-up with CT or endoscopy every 3-6 months is done depending on different patient factors (Figure 10).

Since its introduction by Kanagawa et al, the BRTO has been widely performed as a minimally invasive technique to manage gastric fundal varices. ${ }^{33}$ The technique has an excellent efficacy of $81-100 \%$ and a relapse rate of $0-10 \% .{ }^{(34-39}$ Complications reported include technique related errors like coil migration, balloon malposition and extension of thrombus outside the varices. Renal failure secondary to hemolysis is a known complication with the use of ethanolamine oleate and can be prevented by administration of haptoglobin. Development of ascites, pleural effusion, hepatic encephalopathy and worsening of esophageal varices are also

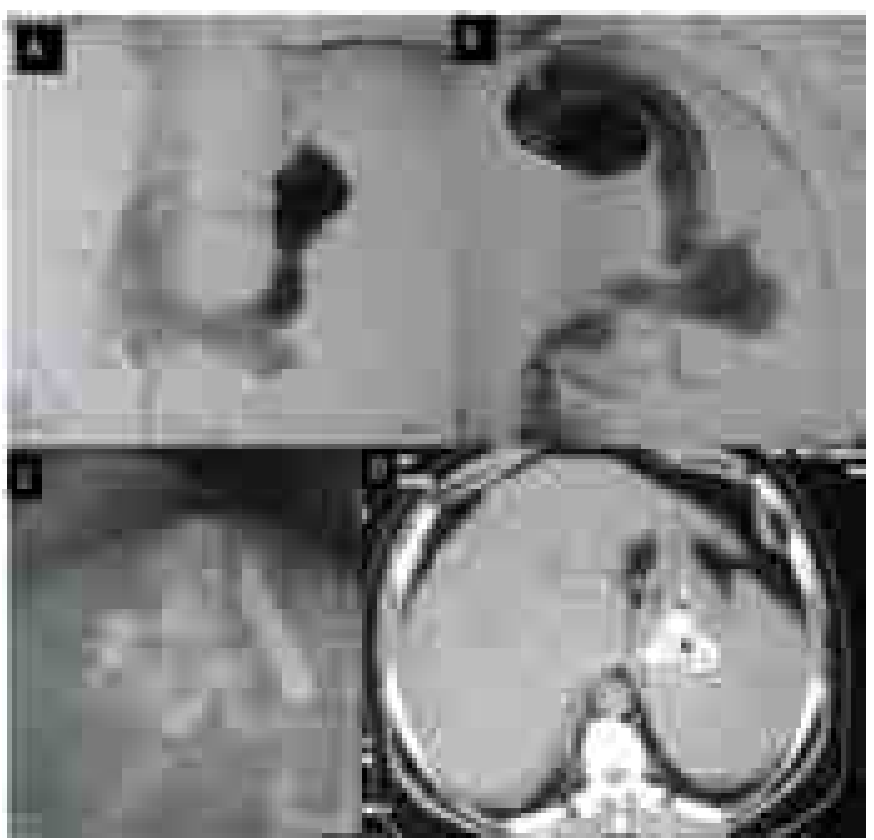

Figure 10: Balloon occluded retrograded transvenous obliteration of gastric varices (BRTO):A\&B) fundic gastric varices are approached through large gastrorenal collaterals of left renalvein,C) sclerosing agent (STS) mixed with lipiodol is injected into the varices with the balloon inflated and is held for three hours,D)follow-up NCCT scan showing lipiodol cast formation within the varices in fundal region of stomach 
observed in occasional patients, depending on their preprocedure functional status. ${ }^{39}$

\section{Occlusion of surgical or radiologic shunts in refractory encephalopathy:}

In occasional patients with advanced cirrhosis the surgical porto-systemic shunts (or TIPS) can cause or aggravate hepatic encephalopathy. In such scenarios therapeutic occlusion of the shunts can be achieved by surgical or endovascular route. The endovascular route is preferred given its minimally invasive nature which is favourable for sick patients. Using the endovascular approach, different occlusion devices are used depending on the size of shunt vessels. Large lumen shunts are closed with larger devices like Amplatzer vascular plugs (Figure 11).

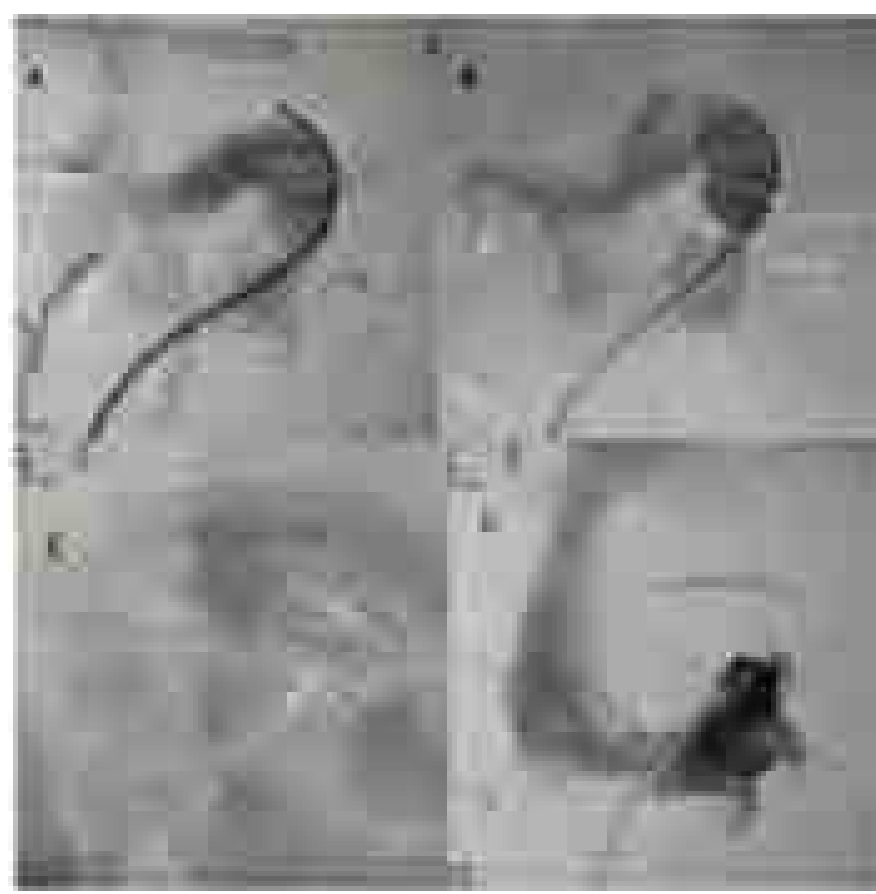

Figure 11: Occlusion of surgical shunts in refractory encephalopathy: A\&B) The surgically created leno-renal shunt for extrahepatic portal vein obstruction (EHPVO) was accessed through the left renal vein. Contrast study showing the large patent shunt, diameter is approximately $11 \mathrm{~mm}, \mathrm{C})$ vascular plug (16 mm diameter) deployed at the level of shunt close to the renal vein, D)post-shunt closure contrast showing complete closure of the shunt and patent left renal vein

\section{References}

1. Sarin SK, Agarwal SR. Extrahepatic portal vein obstruction. Semin Liver Dis. 2002;22:43-58.
2. Al-Busafi SA, McNabb-Baltar J, Farag A, Hilzenrat N. Clinical manifestations of portal hypertension. Int J Hepatol. 2012;2012:203794

3. Reddy SI, Grace ND. Liver imaging. A hepatologist's perspective. Clin Liver Dis. 2002;6:297-310, ix.

4. Ripoll C, Groszmann RJ, Garcia-Tsao G, Bosch J, Grace N, Burroughs A, et al. Hepatic venous pressure gradient predicts development of hepatocellular carcinoma independently of severity of cirrhosis. J Hepatol. 2009;50:923-8.

5. Angermayr B, Cejna M, Koenig F, Karnel F, Hackl F, Gangl A, et al. Survival in patients undergoing transjugular intrahepatic portosystemic shunt: ePTFE-covered stentgrafts versus bare stents. Hepatology. 2003;38:1043-50.

6. Bureau C, Pagan JC, Layrargues GP, Metivier S, Bellot P, Perreault $P$, et al. Patency of stents covered with polytetrafluoroethylene in patients treated by transjugular intrahepatic portosystemic shunts: long-term results of a randomized multicentre study. Liver Int. 2007;27:742-7.

7. Cejna M, Peck-Radosavljevic M, Thurnher SA, Hittmair K, Schoder M, Lammer J. Creation of transjugular intrahepatic portosystemic shunts with stent-grafts: initial experiences with a polytetrafluoroethylene-covered nitinol endoprosthesis. Radiology. 2001;221:437-46.

8. Boyer TD, Haskal ZJ, American Association for the Study of Liver D. The role of transjugular intrahepatic portosystemic shunt in the management of portal hypertension. Hepatology. 2005;41:386-400.

9. Boyer TD. Transjugular intrahepatic portosystemic shunt: current status. Gastroenterology. 2003;124:1700-10.

10. Fisher NC, McCafferty I, Dolapci M, Wali M, Buckels JA, Olliff $\mathrm{SP}$, et al. Managing Budd-Chiari syndrome: a retrospective review of percutaneous hepatic vein angioplasty and surgical shunting. Gut. 1999;44:568-74.

11. Cura M, Haskal Z, Lopera J. Diagnostic and interventional radiology for Budd-Chiari syndrome. Radiographics. 2009;29:669-81.

12. Michl P, Bilzer M, Waggershauser T, Gulberg V, Rau HG, Reiser $\mathrm{M}$, et al. Successful treatment of chronic Budd-Chiari syndrome with a transjugular intrahepatic portosystemic shunt. J Hepatol. 2000;32:516-20.

13. Ochs A, Sellinger M, Haag K, Noldge G, Herbst EW, Walter E, et al. Transjugular intrahepatic portosystemic stent-shunt (TIPS) in the treatment of Budd-Chiari syndrome. J Hepatol. 1993;18:217-25.

14. Koconis KG, Singh H, Soares G. Partial splenic embolization in the treatment of patients with portal hypertension: a review of the english language literature. J Vasc Interv Radiol. 2007; 18:463-81.

15. Alwmark A, Bengmark S, Gullstrand P, Joelsson B, Lunderquist A, Owman T. Evaluation of splenic embolization in patients with portal hypertension and hypersplenism. Ann Surg. 1982;196:518-24.

16. Saugel B, Gaa J, Phillip V, Schmid RM, Huber W. Splenic artery embolization in a woman with bleeding gastric varices and splenic vein thrombosis: a case report. J Med Case Rep. 2010;4:247.

17. Yamakado K, Nakatsuka A, Tanaka N, Fujii A, Isaji S, Kawarada $\mathrm{Y}$, et al. Portal venous stent placement in patients with pancreatic 
and biliary neoplasms invading portal veins and causing portal hypertension: initial experience. Radiology. 2001;220:150-6.

18. Shan H, Xiao XS, Huang MS, Ouyang Q, Jiang ZB. Portal venous stent placement for treatment of portal hypertension caused by benign main portal vein stenosis. World J Gastroenterol. 2005;11:3315-8.

19. Vauthey JN, Tomczak RJ, Helmberger T, Gertsch P, Forsmark C, Caridi J, et al. The arterioportal fistula syndrome: clinicopathologic features, diagnosis, and therapy. Gastroenterology. 1997;113:1390-401.

20. Lopez-Medina A, Peiro J, Gonzalez de Garay M, Antonana MA, Sustacha J, Grande D. Treatment of occluded distal splenorenal shunts with endovascular stents: a report of two cases. Cardiovasc Intervent Radiol. 2001;24:194-7.

21. Lorenz JM, Funaki B, Denison G. Balloon dilatation of a distal splenorenal shunt in a child. AJR Am J Roentgenol. 2005;184:1915-6.

22. underquist A, Vang J. Transhepatic catheterization and obliteration of the coronary vein in patients with portal hypertension and esophageal varices. N Engl J Med. 1974;291:646-9.

23. Bengmark S, Borjesson B, Hoevels J, Joelsson B, Lunderquist A, Owman T. Obliteration of esophageal varices by PTP: a followup of 43 patients. Ann Surg. 1979;190:549-54.

24. Benner KG, Keeffe EB, Keller FS, Rosch J. Clinical outcome after percutaneous transhepatic obliteration of esophageal varices. Gastroenterology. 1983;85:146-53.

25. Lunderquist A, Borjesson B, Owman T, Bengmark S. Isobutyl 2cyanoacrylate (bucrylate) in obliteration of gastric coronary vein and esophageal varices. AJR Am J Roentgenol. 1978;130:1-6.

26. Smith-Laing G, Scott J, Long RG, Dick R, Sherlock S. Role of percutaneous transhepatic obliteration of varices in the management of hemorrhage from gastroesophageal varices. Gastroenterology. 1981;80:1031-6.

27. L'Hermine C, Chastanet P, Delemazure O, Bonniere PL, Durieu JP, Paris JC. Percutaneous transhepatic embolization of gastroesophageal varices: results in 400 patients. AJR Am J Roentgenol. 1989;152:755-60.

28. Sos TA. Transhepatic portal venous embolization of varices: pros and cons. Radiology. 1983;148:569-70.

29. Kwak HS, Han YM. Percutaneous transportal sclerotherapy with N-butyl-2-cyanoacrylate for gastric varices: technique and clinical efficacy. Korean J Radiol. 2008;9:526-33.

30. Tian X, Wang Q, Zhang C, Liu F, Cui Y, Liu F, et al. Modified percutaneous transhepatic variceal embolization with 2octylcyanoacrylate for bleeding gastric varices: long-term followup outcomes. AJR Am J Roentgenol. 2011;197:502-9.

31. Gong GQ, Wang XL, Wang JH, Yan ZP, Cheng JM, Qian S, et al. Percutaneous transsplenic embolization of esophageal and gastriofundal varices in 18 patients. World J Gastroenterol. 2001;7:880-3.

32. Sarin SK, Lahoti D, Saxena SP, Murthy NS, Makwana UK. Prevalence, classification and natural history of gastric varices: a long-term follow-up study in 568 portal hypertension patients. Hepatology. 1992;16:1343-9.

33. Kanagawa H, Mima S, Kouyama H, Gotoh K, Uchida T, Okuda $\mathrm{K}$. Treatment of gastric fundal varices by balloon-occluded retrograde transvenous obliteration. J Gastroenterol Hepatol. 1996;11:51-8.

34. Hirota S, Matsumoto S, Tomita M, Sako M, Kono M. Retrograde transvenous obliteration of gastric varices. Radiology. 1999;211:349-56.

35. Matsumoto A, Hamamoto N, Nomura T, Hongou Y, Arisaka Y, Morikawa $\mathrm{H}$, et al. Balloon-occluded retrograde transvenous obliteration of high risk gastric fundal varices. Am J Gastroenterol. 1999;94:643-9.

36. Koito K, Namieno T, Nagakawa T, Morita K. Balloon-occluded retrograde transvenous obliteration for gastric varices with gastrorenal or gastrocaval collaterals. AJR Am J Roentgenol. 1996;167:1317-20.

37. Kiyosue H, Mori H, Matsumoto S, Yamada Y, Hori Y, Okino Y. Transcatheter obliteration of gastric varices: Part 2. Strategy and techniques based on hemodynamic features. Radiographics. 2003;23:921-37; discussion 37.

38. Ninoi T, Nishida N, Kaminou T, Sakai Y, Kitayama T, Hamuro $\mathrm{M}$, et al. Balloon-occluded retrograde transvenous obliteration of gastric varices with gastrorenal shunt: long-term follow-up in 78 patients. AJR Am J Roentgenol. 2005;184:1340-6.

39. Arai H, Abe T, Takagi H, Mori M. Efficacy of balloon-occluded retrograde transvenous obliteration, percutaneous transhepatic obliteration and combined techniques for the management of gastric fundal varices. World J Gastroenterol. 2006;12:3866-73. 